\title{
Architecture for Organizing Context-Aware Data in Smart Home for Activity Recognition System
}

\author{
Konlakorn Wongpatikaseree, Junsoo Kim, Yoshiki Makino, \\ Azman Osman Lim, and Yasuo Tan \\ School of Information Science \\ Japan Advanced Institute of Science and Technology, Ishikawa, Japan 923-1211 \\ \{w.konlakorn, junsoo, m-yoshi, aolim, ytan\}@jaist.ac.jp
}

\begin{abstract}
Knowing human activity in each day is relevant information in several purposes. However, existing activity recognition systems have limitation to identify the human activity because they cannot get the appropriate information for recognition. To address this limitation, we present three relevant components in Context-aware Activity Recognition Engine (CARE) architecture for organizing context-aware information in home. First, we introduce Context Sensor Network (CSN). The CSN provides the raw environment information from the diversity of sensors. Second, data manager component is proposed to process the pre-processing in the raw data from the CSN. The data must be normalized and transformed in order to make the system more efficient. The last component is system repository that composes of three essential tasks for controlling the information in the system. In this paper, the ontology based activity recognition (OBAR) system is used to evaluate the data from proposed components. The high accuracy of results can refer to the well organization of proposed components.
\end{abstract}

Keywords: Human activity, context-aware activity recognition engine, ontology based activity recognition.

\section{Introduction}

Recently, the high technologies play vital roles to built a variety of the healthcare system, especially in smart home. For example, home health care (HHC) system 1 proposed to help the people gain the better health in the home. Nevertheless, only home user's health condition might not enough for analysing and treatment the disease because sometime home user might perform something that leads to the disease, but home user does not know by himself or herself.

In that sense, human activity recognition system has been proposed to capture the human activity in each day. The physician or healthcare system can utilize the results from the activity recognition system for diagnosis, treatment or prevention the disease. However, to observe the human activity is not an easy task because there is several environment information in home that we have to consider such as home environment or human information. For sensing data in 
the home, most of the researches have implemented the system that obtains the context information based on only single concept: body sensor network (BSN) or home sensor network (HSN). The system might get the imperfect data 2 if only one concept is developed. It can lead to occur the "Ambiguous activity problem". In this paper, we aim to improve the ability of activity recognition by proposing three relevant components in the Context-aware Activity Recognition Engine (CARE) architecture that can organize and provide the appropriate information for recognition the human activity.

The rest of paper is organized as follows: in section 2, we briefly describe related works on activity recognition area, especially in sensing data process. Then, we introduce the overview of CARE system architecture in section 3 . Section 4, CSN proposes for collecting the environment information in home. After that, we present the way to normalize the data in section 5. In section 6, System repository component shows the process for controlling the information in the system. Next, in section 7 , we demonstrate the experiment and results in this research. Finally, conclusion and future work are in section 8 .

\section{Background and Related Worked}

Among of existing researches in activity recognition area, they have proposed several ways to identify the daily physical activity. Not only the classification process plays a vital role for recognition the human activity, but sensing data process is also relevant to collect the appropriate information. Thus, in this section, we will describe the existing techniques that used for sensing data in activity recognition system. Currently, there are three main sensing data techniques.

First, visual sensing technique has been proposed in the computer vision area. To perceive the information in this technique, high-resolution camera is mainly used for collecting the image or video files. Normally, there are two ways to perceive data: single viewpoint-based surveillance and multi viewpoint-based surveillance. In the single viewpoint-based surveillance, it is not difficult to design the system architecture because data from only one camera is used to recognize the human activity [3], but in the multi viewpoint-based surveillance method [4, it has the heavy task to perform the camera network for synchronisation the information between cameras. The information from distributed camera network can be used to identify the human in several purposes such as human action, human location, or travel time in each room. However, the privacy problem is the crucial problem for classification the human activity.

The rest two techniques for sensing data are BSN and HSN. The concept of BSN [5] is that attach the sensor on the human body for capturing the motion of human. Thus, system will perceive only the information from the human sensor. Nevertheless, only information from the human sensor might not enough to recognize some specific activities such as "Watching TV", or "Working on computer". The HSN has been proposed for solving in this problem. Diversity of sensors is embedded in the home facility for detecting the interaction between human and object. The system recognizes the human activities by monitoring, 


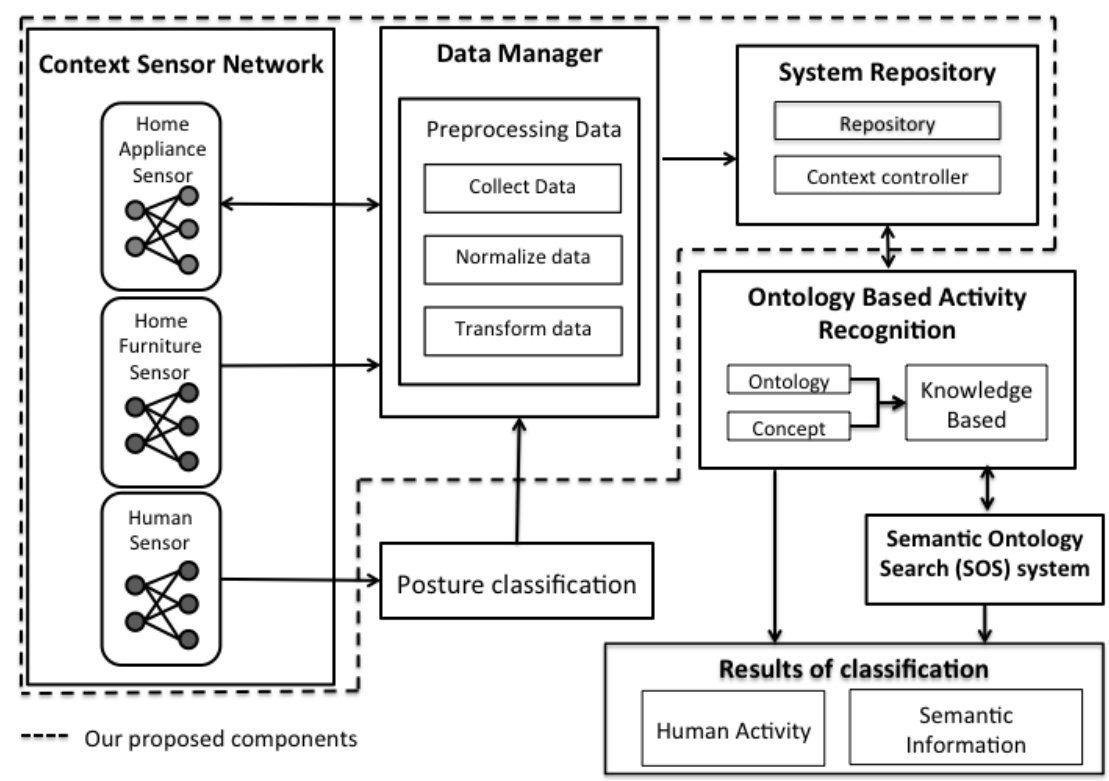

Fig. 1. CARE Architecture

which home facility is being used and how long user spends time on that facility 6]. However, the drawback of this technique is when several objects are activated in the same time. It leads the system classify several possible resultant activities.

\section{CARE Architecture Overview}

Until now, homes in $21^{\text {st }}$ have embedded the ubiquitous system for different purposes. Sensing, managing, controlling the amount of data in smart home are a challenging task for organizing proper data before processing the activity recognition. According to the dis/advantage of sensing data techniques in section 2 , designing the architecture is effected to the results of activity recognition system. In this section, we briefly describe the system architecture that used for organization the home information and human information for activity recognition. Figure. 1 presents a high-level of CARE architecture. In our proposed, there are tree main components for obtaining, managing, and controlling the data in CARE architecture. First, Context Sensor Network (CSN) observes the surrounding information in smart home, including human information (Section 4). Second, data manager normalizes the raw data from the CSN (Section 5). Last, system repository takes a responsibility to control the flow of data in the system (Section 6). 


\section{Context Sensor Network (CSN)}

To obtain the relevant information in the real environment, CSN is proposed with diversity of sensors and protocols. The CSN is a sensor network that typically uses for collecting the context data in the smart home. Our testbed of this paper is establishing in an iHouse 7]. The iHouse is designed for development of the next-generation home network system. Two floors with $107.76 \mathrm{~m}^{2}$, more than 250 sensors and home appliance are connected through ECHONET, UPnP, and Zigbee. Considering in the home environment, there are several details that we have to consider. Two of relevant information for classifying the human activity in the home are home environment information and human information. There are three kinds of sensor networks in CSN as followed:

\subsection{Home Appliance Sensor Network}

The main task of home appliance sensor network is to capture the use of home appliance. Diversity of sensors is built into the home appliance in iHouse. Most of home appliance can be detected by measure the change of electric current from the power consumption. Meanwhile, water-flow sensors are embedded in the smart home for monitoring the use of water appliance such as sink, shower, or flushing. This kind of information is relevant for classifying the specific activity such as "Watching TV", "Working on computer" or "Cooking". For example, we can know the user might performing the "Cooking" activity when the system recognize "Electric stove" is being used, or the system classify the activity as "Taking a bath" if the water-flow sensor detects the flow of water from the "Shower" object.

In our research, to transfer the data in home appliance sensor network, there are two protocols for sending the requested command to each sensor: ECHONET 8] and UPnP 9. The ECHONET is an international home network protocol standard that is used to control, monitor, and gather information from equipment, and sensor. UPnP is used to make the requested command for monitoring the sensor status. An interval time is set to five seconds for sending the requested command.

\subsection{Home Furniture Sensor Network}

Apart from the home appliances, we still need to consider other home environment, such as "Sofa", "Bed", or "Broom" object. Normally, the object in this sensor network can be divided into two cases. First is the direct purpose object. That means the object can infer to only one activity. For example, "Broom" object can be used only for "Sweeping the floor" activity. Second is the multipurpose object. There is some home furniture in home that the user uses in several purposes. For example, home user uses "Sofa" object for sitting and watching TV, or lying down on the "Sofa" for relaxing.

This kind of home furniture information can compose with the home appliance information for performing the specific activity. For example, if "Computer" object is turned on, it does not means that user performing "Working on computer" 


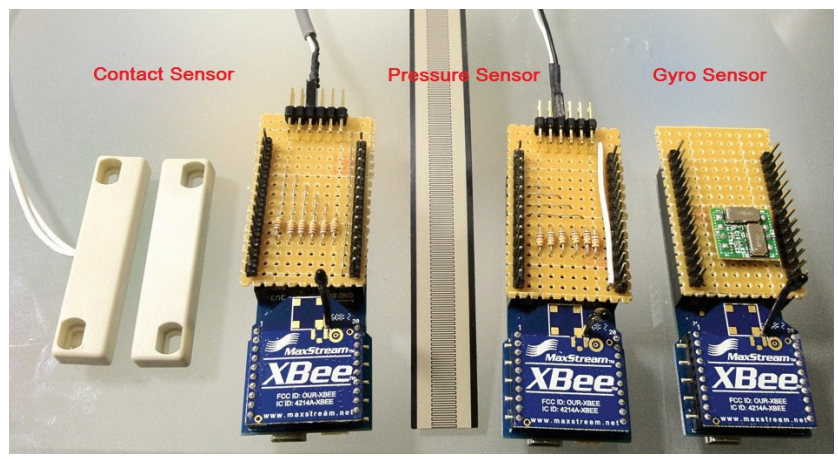

Fig. 2. Arduino Fio with external board and sensor

activity. Normally, user tends to sit on the "Chair" for "Working on computer". In this paper, pressure sensor, gyro sensor, and magnetic sensor are deployed and attached on the home furniture. For example, pressure sensors are attached on the "Bed" object to detect the "Bed" is being used or not. Gyro sensor is attached on the home furniture that has movement such as "Broom", "Mop", or "Coffee bottle". Magnetic sensor is used for the item that has open-close property such as "Cupboard".

Nevertheless, we cannot utilize the ECHONET protocol for transmission the data same as home appliance sensor network because most of the home furnitures are not in the international home network protocol standard. In this sensor network, we select the Arudino board [10] for sensing the home furniture data. There are two basic module in this sensor network: sensor node and coordinator. For the sensor node, we have selected the Arudino Fio as a microcontroller board based on the ATmega328P. Arduino Fio is connected with pressure sensor, gyro sensor, and magnetic sensor via external board, shown in Fig. 2 Arduino Fio also allows the developer to program it wirelessly, over a pair of XBee radio. Therefore, we emulated the Zigbee protocol for transmission the data between home furniture sensor and server. For the coordinator side, Arduino Ethernet is developed belonging to Xbee shield. The Xbee shield allows an Arduino board to communicate wirelessly using Zigbee. At this stage, the coordinator node will collect data from sensor node via Zigbee protocol and transmit to server via Ethernet cable. Each coordinator node will be placed on the conner of each room. Therefore, system will perceive where the data is come from based on the "Coordinator id" and "Sensor id".

\subsection{Human Sensor Network}

Normally, only the home environment data might not enough to conform the user's context for classifying the human activity. It can lead to the "Ambiguous activity problem" when using only home environment data. For example, if we consider only object activation to perform the activity recognition system, the 
system cannot guarantee the high accuracy when several object are being used. It makes the system generated several possible resultant activities. Therefore, the human sensor network is used to monitor the human information such as human location. Infrared sensors are deployed in each room in iHouse for detecting the human location. The current human location is relevant to activity recognition because it can give useful hints about which activities s/he can or cannot perform.

Nonetheless, using human location and object activation for classification are still encountering the "Ambiguous activity problem" because sometime human location does not hint any activities if several objects are being used in the same current human location. Recently, the other human information are introduced for improving the activity recognition. For example, human posture information is utilized with the common user's context (object activation and human location) [1]. It shows the advantage to reduce the "Ambiguous activity problem" when using human posture. To obtain human posture in CARE architecture, range-based algorithm [12] is proposed to classify the human posture based on a range between body parts. The devices that are capable to measure the distance between sensors are used in this architecture.

\section{Data Manager}

According to the CSN, there are several environment data in home that the system have to obtain because several objects in the home can infer to the human activity. Not only the amount of data in smart home is effect to the system, but also the perfect of data. Although the system can obtain the data from the diversity of sensor, it still suffers with missing data or noise from the sensor. There are particular problems in data when due with hardware. Therefore, data manager component aim to normalize data before send to the system repository.

There are two techniques to normalize the raw data from the CSN. Firstly, supply missing data function is developed for solving the missing data problem. The supply missing data function will find and add the suitable data for making the user's context perfectly. For example, the system cannot perceive the human location from the infrared sensor if the user has little movement. In this case, the supply missing data function will retrieve the last human location instead the current human location for making the completed user's context. Second, eliminate data function is the relevant function to cut the unexpected data or noise from the sensor. The unexpected data problem can make the result of recognition changed easily. In this paper, we apply the threshold technique in eliminate data function. The lower bound is set for filtering the noise in some circumstances. For example, normally, although we turn off the TV but plugging, the sensor still detect a little electric current from TV. It can make the system decide that TV is being used. The result might be changed easily if there is this kind of noise. 


\section{System Repository}

To control the data in the CARE architecture, the system repository play an important role to control data flow between system repository and OBAR, illustrated in Fig. 3 The system repository can be divided into two modules. First, repository is the module that takes a responsibility to keep the normalized data from the data manager, and also store the temporal reasoning from the OBAR. Second, context controller module has a duty to control all of data in the system. There are three main tasks in context controller:

- Mapping Data: Based on CARE architecture, ontology concept is used to explicit the huge information from the variety of sensors. In this research, the ontology concept is described in the abstract level. The ontology application management framework [13] is adopted to map between the properties concept in ontology and the data in the repository. However, we cannot use a relational database through this step directly. We need to transform the relational database to resource description framework (RDF) for interchange the data on the web.

- Composition Data: The necessary information in the repository will be conform to the user's context. In one user's context, system can know various kind of semantic information such as object activation, human location, time or human posture. The user's context will be served as the input data in OBAR. Every one minute, system will conform the user's context for classifying the human activity.

- Reprocessing Data: According to the original idea of ontology concept, it does not support the temporal reasoning. In this task, we implement the external java program to keep tracking the temporal reasoning. Then the temporal reasoning will be collected in the repository, and it is sent to the inference method for the next classification. This information is vital useful when system lacks data to classify the human activity.

\section{$7 \quad$ Experiment and Results}

\subsection{Ontology Based Activity Recognition (OBAR)}

To evaluate the organization's performance of the three proposed components, we implemented the OBAR [1] for inference the human activity based on data from proposed components. In this section, we will describe the workflow of OBAR briefly. There are two parts of OBAR: ontology modeling and recognition engine. For modeling the human activity, the ontology concept is used to explicit the semantic information in smart home. There are two main ontologies models. First, the context-aware infrastructure ontology is designed for definition the surrounding information in smart home. Second, the activity log ontology is used to identify the historical information. As describe in section 6, these two ontologies will be mapped with the repository by context controller. 


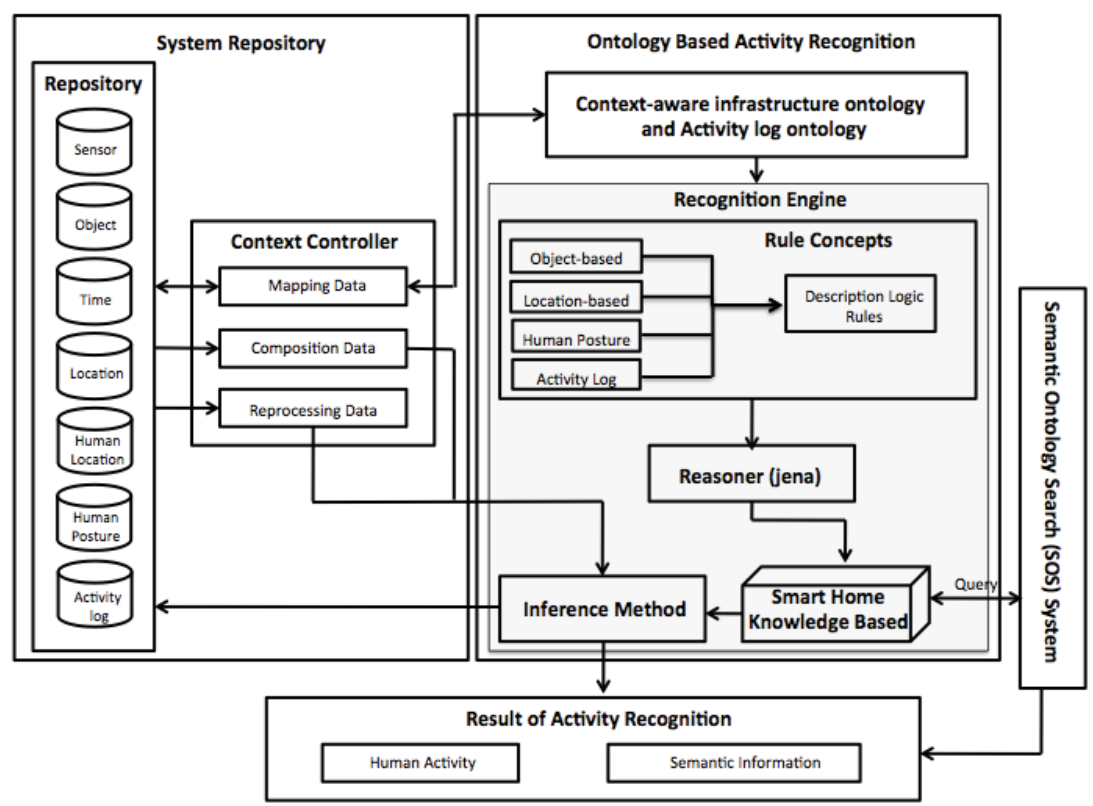

Fig. 3. Data flow between system repository and OBAR

Recognition engine is the core of OBAR to classify the human activity. Normally, the ontology concept does not have the ability to classify the human activity. Description logic (DL) rules are established for supporting inference and reasoning. To create the DL rules, object activation, human location, human posture, and activity log are used to create the DL rules. The example below indicates the DL rule for "Washing dishes" activity.

Wash dishes $\sqsubseteq$ Functional Activity

$\sqsubseteq$ Kitchen Activity

$\sqcap$ use(Object.Furniture $($ Sink $))$

$\sqcap$ Object.Human.Current_location(kitchen)

$\sqcap$ HumanPosture (Stand)

$\sqcap$ LastActivity.Kitchen Activity(Eating or drinking)

After that, the built-in reasoner is implemented for computing the DL rules for the new knowledge and collect into the smart home knowledge based. Inference method has a responsibility to check the data from the composition data that consistent with the knowledge in smart home knowledge based or not. The system will give the results if user's context is consistent with the rule in smart home knowledge based. After that, the system will keep the results of classification into the repository for processing in the next classification. 
Table 1. Recognition accuracy of OBAR

\begin{tabular}{|l|l|l|}
\hline Activity & Accuracy & Other possible resultant activities \\
\hline$A_{1}=$ Working on computer & $92.25 \%$ & $A_{2}(6.90 \%), A_{7}(0.42 \%), A_{14}(0.43 \%)$ \\
\hline$A_{2}=$ Watching TV & $97.94 \%$ & $A_{14}(2.06 \%)$ \\
\hline$A_{3}=$ Reading a book & $100 \%$ & - \\
\hline$A_{4}=$ Scrubbing the floor & $93.75 \%$ & $A_{14}(6.25 \%)$ \\
\hline$A_{5}=$ Sweeping the floor & $96.67 \%$ & $A_{14}(3.33 \%)$ \\
\hline$A_{6}=$ Sitting on the toilet & $93.75 \%$ & $A_{1}(2.08), A_{14}(4.17 \%)$ \\
\hline$A_{7}=$ Taking a bath & $100 \%$ & - \\
\hline$A_{8}=$ Lying down \& relaxing & $100 \%$ & - \\
\hline$A_{9}=$ Sleeping & $91.86 \%$ & $A_{3}(3.49 \%), A_{14}(4.65 \%)$ \\
\hline$A_{10}=$ Making coffee & $100 \%$ & - \\
\hline$A_{11}=$ Cooking & $86.21 \%$ & $A_{14}(13.79 \%)$ \\
\hline$A_{12}=$ Eating or drinking & $100 \%$ & - \\
\hline$A_{13}=$ Washing dishes & $100 \%$ & - \\
\hline$A_{14}=$ Idle & $100 \%$ & - \\
\hline
\end{tabular}

\subsection{Results and Discussion}

Table1illustrates the recognition accuracy in 14 activities. The recognition accuracy of OBAR exhibits the organization's performance of proposed components. Although OBAR can achieve the high accuracy with $96.6 \%$, it still has problems that make the OBAR classify incorrect results.

The delay of sensor in CSN and the interval time for composition data in system repository (composition data task) are not synchronized. The strange result ("Taking a bath" activity) in the "Working on computer" is the outstanding example that shows the asynchronous problem. Since when sensor is activated, it will be delayed one minute for sensing the next status while the context controller will compose the user's context every one minute. Consequently, it is possible that the user already changes the activity, but the system still using the old information for classification. To address these shortcoming, in the future work, the interval time for composition data should have to have the adaptive function to adjust the interval time for composition the data for recognition the human activity.

\section{Conclusion}

In this paper, we proposed the three components in CARE architecture that use for organizing context-aware data in smart home. First, we introduced the CSN that used to observe the surrounding information in the smart home. Three kinds of sensor networks are proposed for collecting the object activation and human information via diversity of sensors and protocols. Second, data manager component is presented for normalizing the data. This component is helpful for the system to make the complete data. Supplying missing data function and eliminate noisy data function are implemented for providing the appropriate 
data to the system repository. Last, system repository is exhibited the vital role to control the huge of data in the system. Based on the real data from these three components, the OBAR can achieve the high recognition accuracy with 96.60 $\%$. Even though the results cannot exactly conclude that proposed components make the high recognition accuracy, it demonstrates that proposed components can provide the appropriate information for the classification.

\section{References}

[1] Takahashi, S., Maeda, S., Tsuruta, N., Morimoto, T.: A home health care system for elderly people. In: Proceedings of the 7th Korea-Russia International Symposium on Science and Technology, KORUS 2003, Pohang, vol. 2, pp. 97-102 (July 2003)

[2] Henricksen, K., Indulska, J.: Modelling and using imperfect context information. In: Proceedings of the Second IEEE Annual Conference on Pervasive Computing and Communications Workshops, Washhington, DC, pp. 33-37 (March 2004)

[3] Ben-Arie, J., Wang, Z., Pandit, P., Rajaram, S.: Human activity recognition using multidimensional indexing. IEEE Transactions on Pattern Analysis and Machine Intelligence 24(8), 1091-1104 (2002)

[4] Fiore, L., Fehr, D., Bodor, R., Drenner, A., Somasundaram, G., Papanikolopoulos, N.: Multi-camera human activity monitoring. Journal of Intelligent and Robotic System 52(1), 5-43 (2008)

[5] Maurer, U., Smailagic, A., Siewiorek, D., Deisher, M.: Activity recognition and monitoring using multiple sensors on different body positions. In: International Workshop on Wearable and Implantable Body Sensor Networks, Boston, pp. 113116 (April 2006)

[6] Zhang, S.S., McClean, Scotney, B., Chaurasia, P., Nugent, C.: Using duration to learn activities of daily living in a smart home environment. In: 4th International Conference on Pervasive Computing Technologies for Healthcare (PervasiveHealth), Munich, pp. 1-8 (March 2010)

[7] Tan, Y.: Home Network Technologies for Smart Houses. Impress R\&D (2011) (in Japanese)

[8] Matsumoto, S.: Echonet: A home network standard. IEEE Pervasive Computing 9(3), 88-92 (2010)

[9] Kim, K.S., Park, C., Seo, K.S., Chung, I.Y., Lee, J.: Zigbee and the upnp expansion for home network electrical appliance control on the internet. In: The 9th International Conference on Advanced Communication Technology, Gangwon-Do, vol. 3, pp. 1857-1860 (February 2007)

[10] Arduino, http://www.arduino.cc (accessed: February 18, 2013)

[11] Wongpatikaseree, K., Ikeda, M., Buranarach, M., Supnithi, T., Lim, A., Tan, Y.: Activity recognition using context-aware infrastructure ontology in smart home domain. In: Seventh International Conference on Knowledge, Information and Creativity Support Systems (KICSS), Melbourne, pp. 50-57 (November 2012)

[12] Wongpatikaseree, K., Lim, A., Tan, Y., Kanai, H.: Range-based algorithm for posture classification and fall-down detection in smart homecare system. In: IEEE 1st Global Conference on Consumer Electronics (GCCE), Tokyo, pp. 243-247 (October 2012)

[13] Buranarach, M., Thein, Y., Supnithi, T.: A community-driven approach to development of an ontology-based application management framework. In: The 2nd Joint International Semantic Technology Conference (JIST), Nara (December 2012) 